\title{
A Study of Sedentary Behaviour in the Older Finnish Twin Cohort: A Cross Sectional Analysis
}

\author{
Maarit Piirtola, ${ }^{1,2}$ Jaakko Kaprio, ${ }^{1,3,4}$ and Annina Ropponen ${ }^{5}$ \\ ${ }^{1}$ Department of Public Health, Hjelt Institute, University of Helsinki, P.O. Box 41 (Mannerheimintie 172), 00014 Helsinki, Finland \\ ${ }^{2}$ UKK Institute, Centre for Health Promotion Research, Tampere, Finland \\ ${ }^{3}$ Department of Mental Health and Substance Abuse Services, National Institute for Health and Welfare, \\ P.O. Box 30 (Mannerheimintie 166), 00300 Helsinki, Finland \\ ${ }^{4}$ Institute for Molecular Medicine (FIMM), University of Helsinki, P.O. Box 20 (Tukholmankatu 8), 00014 Helsinki, Finland \\ ${ }^{5}$ Finnish Institute of Occupational Health, Topeliuksenkatu 41 aA, 00250 Helsinki, Finland \\ Correspondence should be addressed to Maarit Piirtola; maarit.piirtola@helsinki.fi
}

Received 12 November 2013; Revised 17 February 2014; Accepted 6 March 2014; Published 15 April 2014

Academic Editor: Meike Bartels

Copyright (c) 2014 Maarit Piirtola et al. This is an open access article distributed under the Creative Commons Attribution License, which permits unrestricted use, distribution, and reproduction in any medium, provided the original work is properly cited.

\begin{abstract}
The aim of the study was to investigate the effects of age, sex, and body mass index (BMI) on total sitting time among the Finnish twin cohort. Also, heritability and environmental factors were analysed. The final sample included 6713 twin individuals 53-67 years of age (46\% men). Among them there were 1940 complete twin pairs (732 monozygotic [MZ] and 1208 dizygotic [DZ] twin pairs). Sedentary behaviour was queried with a self-reported questionnaire with multiple-choice questions about sitting time at different domains. The mean total sitting time per day was 6 hours 41 minutes (standard deviation: 2 hours 41 minutes). The total sitting time was less in women than in men $(P=0.002)$. Older age was associated with less total sitting time $(P<0.001)$. Those with higher body mass index had higher total sitting time in age and sex adjusted analysis $(P<0.001)$. MZ pairs were more similar for sitting time than DZ pairs, with initial estimates of heritability for the total sitting time of 35\%.The influence of shared environmental factors was negligible (1\%), while most (64\%) of the variation could be ascribed to unique environmental factors, the latter including measurement error.
\end{abstract}

\section{Introduction}

Sedentary behaviour, measured as sitting time, is one of the major global public health concerns $[1,2]$. A high amount of sitting is independently associated with overweight $[3,4]$ and cardiometabolic risk [4]. In addition, a high amount of sitting time increases all-cause and cardiovascular diseaserelated mortality independent of whether a person is meeting physical activity guidelines [5-7]. Thus, actions to investigate the backgrounds and genetics of sedentary behaviour need to be studied further in order to implement effective preventive actions [8].

Sedentary behaviour has been defined in various ways [9]. It has been suggested that sedentary behaviour can be a paradigm of its own, distinctive to that of moderate- to vigorous-intensity physical activity, with independent effects on health [2]. Thus, sedentary behaviour is not simply the absence of moderate- to vigorous-intensity physical activity [2] or even the presence of light physical activity [9]. Recently one of the globally accepted suggestions for the use of the term sedentary behaviour had been given by the Sedentary Behaviour Research Network, including the definition of sedentary behaviour "as any waking behaviour characterized by an energy expenditure $\leq 1.5$ metabolic equivalents (METs) while in sitting or reclining posture" [10]. The Network also suggests using the term inactive in describing "those who are performing insufficient amount of moderate- to vigorousintensity physical activity (MVPA) (i.e., not meeting specific physical activity guidelines)" [10].

In addition to variation in definitions, sedentary behaviour can also be measured in various ways. Even though there is a recommendation that sedentary behaviour should be 
monitored by incorporation of both self-reported and devicebased measures [11], no consensus exists for a golden rule of method to measure sedentary behaviour [11].

In the observational research, sedentary behaviour has been investigated with self-report questions about sitting time during different sedentary activities such as watching TV or using the computer and sitting at work or in vehicles $[12,13]$. Especially in large population-based studies, those involving thousands of participants, the self-reported sitting time is a useful method despite its inaccuracy of the total amount of physical activity and the potential recall bias [11].

Understanding why some people are physically active and others inactive or behaving in a sedentary way is important in planning public health interventions [14]. It has been shown that age, health status, self-efficacy, and motivation in addition to social and physical environmental factors are associated with physical activity levels [14]. In two recently published Finnish population-based studies using self-administered questionnaires, the mean sitting times per day in men and women 25 to 60 years of age varied between 5.5 to 6.9 hours and 5.2 to 6.4 hours, respectively $[15,16]$. The total sedentary times in these Finnish cohorts $[15,16]$ were less than reported in a large multiethnic cohort study with over 130,000 older subjects where the average daily sitting time was 8.0 hours in men and 8.2 in women [7]. It has been reported that differences in sitting times are based on ethnicity, age, educational level, and body mass index (BMI) [17].

Another potential factor related to physical activity, inactivity, and sedentary behaviour is family background and genetic predisposition [18]. It has been shown that when exercise participation is determined as 60 minutes/week at a minimum intensity of $4 \mathrm{METs}$, interindividual genetic differences accounted for $48-71 \%$ of variance in both sexes [19]. A recently published review presented a variance for genetics that was widely spread, as heritability estimates ranged from 0 to $85 \%$ for physical activity and from 25 to $60 \%$ for physical inactivity depending on definitions of physical activity levels, population, age, and other differences between studies [18]. Among adolescents, genetics has explained $72 \%$ to $85 \%$ of variance in exercise participation determined as sedentary, moderate, or vigorous exercise by METs [20]. The contribution of genetic factors to variation in sedentary behaviour frequency per week among 12-20 year-old boys was $35 \%$ to $48 \%$ and $19 \%$ to $34 \%$ among same aged girls [21]. In addition, the influence of shared environmental factors decreased along age in adolescence [21]. In another study, the role of genetics in MVPA was 59\% among adolescents and $12 \%$ in young adults [22]. In adolescents, genetics explained $34 \%$ of the variance of sedentary time (per week), whereas shared environmental (household) explained $10 \%$ and unique environmental factors $56 \%$ of the variation [22]. The influence of genetics in sedentary behaviour in young adults was $28 \%$ of variance suggesting a somewhat increasing impact of unique environmental factors along age. In older adult twins, genetics explained $31 \%$ of the time spent in sedentary behaviour ( $\leq 1.5 \mathrm{METs})$ whereas it was larger $(47 \%)$ for MVPA [23]. The effect of unique environment (i.e., the exposures and experiences mainly related to adulthood) was
$52-55 \%$ of variance [23]. It is possible that genetic influences are different in inactivity and sedentary behaviours compared to physical activity but there is an inconsistency between previously published results $[18,23]$.

In this paper, the focus was to describe the latest data collection of physical activity and sedentary behaviour in the Finnish twin cohort $[24,25]$. The main aim was to investigate the relative contribution of genetics and environmental factors of sedentary behaviour in 53 to 67 year-old men and women from the Finnish twin cohort study. In addition, the effects of age, sex, and body mass index (BMI) on total sitting time were investigated.

\section{Materials and Methods}

2.1. Participants. The population-based data of the older Finnish twin cohort was available for 16,269 same-sex twin pairs [26]. The extensive longitudinal data includes four waves of surveys (baseline in 1975 and three follow-up surveys in 1981, 1990, and 2011/12) [24-26]. During the fourth wave (October 2011 to June 2012) all twins born 1945-1957 ( $n=11$ 738 ) originally identified to the cohort in 1974 and known to be alive in 2011 with an address in Finland were sent the questionnaire survey, either in Finnish or in Swedish. All subjects, except 13 individuals, had answered at least one of the earlier surveys (1975 and/or 1981 and/or 1990) along the follow-up. The vital status of the original cohort members was updated in 2011 from the national Finnish population register. All surviving twins received the survey irrespective of their cotwin's status. The data collection of the fourth wave is mainly described in the review article in 2013 [25]. The protocol was designed and performed according to the principles of the Helsinki Declaration and was approved by the Ethical Committee of the Helsinki University Central Hospital.

2.2. Methods. To maintain continuity the original questions (such as in 1975 and 1981) were used wherever possible, despite the development of better measurements for some topics. The questionnaire of the fourth wave included comprehensive questions about self-reported health, functional capacity, and lifestyle factors, described in more detail in the review published in 2013 [25].

2.2.1. Physical Activity. All four surveys included questions about the quality and quantity of leisure-time physical activities: amount (per year), duration (per one session), frequency (per month), and intensity of leisure-time physical activity as well as daily time of commuting by physically active means (including walking, jogging, and cycling) to and from work (minutes per day) (see Appendix A). Also physical workload of the present or previous work was asked.

2.2.2. Sedentary Behaviour. Sedentary behaviour has been queried in the fourth questionnaire with multiple-choice questions about sitting time during different activities (see Appendix B). The participants have answered how many hours, on average, they are sitting per day: (1) in office or 
similar places, (2) at home watching TV or videos, (3) at home at the computer, (4) in a vehicle, and (5) elsewhere. Each question had four alternatives: (a) less than an hour, (b) an hour-less than two hours, (c) two hours-less than four hours, and (d) four hours or more. We assumed the intensity of each of these sedentary activities to be no more than 1.5 METs [9]. Total daily sitting time was the sum of the midpoints of the specific sitting categories, using 30 minutes for "less than an hour," 90 minutes for "an hour-less than two hours," 180 minutes for "two hours-less than four hours", and 300 minutes for "four hours or more." For those twin individuals ( $n=4034$ ) who were not working at the moment of the survey, sitting time at work was denoted as zero minutes.

2.3. Statistical Methods and Data Analysis. The total sitting time parameter has been developed based on five sitting activities by developing a summary variable (sitting time in hours and/or in minutes). Only those twin individuals reporting sitting times in all five sitting domains, including those not at work with zero sitting time on that item, were chosen for the final analyses. The normality of the sitting summary variable was tested with Kolmogorov-Smirnov and Shapiro-Wilk tests. The total sitting sum variable had the skewness value of 0.676 and kurtosis of 0.345 indicating that sitting time was not fully, normally distributed $(P<0.001)$ (Figure 1). However, tests of normality are extremely robust [27] and our relatively large sample size will result in a minor departure from normality being significant. Furthermore, the methods used in analyzing twin data are robust to minor deviation of normality. The original cohort had been a sample of twin pairs and that was taken into account, and robust standard errors were derived to obtain correct confidence intervals and $P$ values [28]. The chi-squared test, Spearman correlation, and independent-sample $t$-test were used in the descriptive analyses. In the linear regression models, with 95\% confidence intervals (95\% CI), the effects of sex and age were analysed together. Age was used as a continuous parameter in the analyses.

Body mass index (BMI) was calculated by individual's weight and height $\left(\mathrm{kg} * \mathrm{~m}^{-2}\right)$. BMI values 20 or less as well as BMI 36 or more were combined to be the first and the last categories for descriptive purposes. The sex and age adjusted linear regression model with $95 \%$ confidence intervals was used in analysing the association between the total sitting time and BMI.

To investigate the heritability of physical activity, the phenotype was assumed to have an underlying, continuous liability. Heritability was analysed by calculating pairwise correlation coefficients by zygosity and sex and further comparing the results of monozygotic twins (MZ) to samesex dizygotic twins (DZ). As MZ twins are genetically alike, that is, share the same genomic sequence, while DZ twins share on average $50 \%$ of the their segregating genes, increased similarity of $\mathrm{MZ}$ pairs versus $\mathrm{DZ}$ pairs is taken as evidence for the presence of genetic effects. In addition, Falconer's formula [29] was used to calculate the proportion of variance estimated by the ratio of $\mathrm{MZ}$ and $\mathrm{DZ}$ twin correlations explained by additive genetics $\left(h^{2}=2\left(r_{\mathrm{MZ}}-r_{\mathrm{DZ}}\right)\right)$, shared environmental factors $\left(c^{2}=2 r_{\mathrm{DZ}}-r_{\mathrm{MZ}}\right)$, and unique environmental factors $\left(e^{2}=1-h^{2}-c^{2}\right)$ based on $\mathrm{MZ}$ correlations being twice that or less compared to DZ correlations.

All analyses were performed with the Stata version 12 or the IBM SPSS version 21. In all analyses, significance was considered to be $P<0.05$.

\section{Results}

The fourth questionnaire was returned by 8406 twin individuals (3750 men, 4656 women) resulting in a response rate of $72 \%$. Complete data of sedentary behaviour (those answered in all five sitting domains) was available for 6713 participants (3082 men, 3631 women). The data covered $80 \%$ of those who returned the questionnaire.

There were more women (61\% versus $54 \%, P=0.009$ ) in those having missing information at least in one sitting domain compared with those with information in all five domains. Those with any missing information were older (mean age 62 years versus 60 years, $P=0.000$ ), not working full time ( $81 \%$ versus $41 \%, P=0.000)$, and they were more likely obese, $\mathrm{BMI}>30$ (19.1\% versus $16 \%, P=0.009)$. There were no significant differences in the amount of leisuretime physical activity between those with or without missing information about their sitting times $(P=0.068)$.

The final analysis sample $(n=6713)$ comprised 310 complete monozygotic male pairs (MZM), 422 monozygotic female pairs (MZW), 527 dizygotic male pairs (DZM), and 681 dizygotic female pairs (together 1940 pairs). The average age of the twins was 60 years (range 53 to 67 years) in both sexes and their mean BMI was 26 (range 15 to 48 ). At the time of the survey, $59 \%$ of the twin individuals reported working full time. The physical workload of the present work was mainly sedentary work in $38 \%$ of the twins; $12 \%$ of twins had work which involved standing and walking but no other physical activity; $41 \%$ had work which in addition to standing and walking required lifting and carrying; and $8 \%$ did heavy physical work, whereas $1 \%$ of the twins reported a mixed combination of all these kinds of work loading conditions.

The mean sitting time per day was 6 hours 41 minutes (SD: $2 \mathrm{~h} 41$ minutes) (Figure 1). In men, the mean total sitting time was 6 hours $46 \mathrm{~min}$ (SD: $2 \mathrm{~h} 50$ minutes) and in women 6 hours 34 minutes (SD: 2 hours 34 minutes). One quarter of the twins reported sitting 4.5 hours or less per day, a half $4.5-$ 6.5 hours a day, and $10 \%$ at least 10.5 hours a day. The sitting times during different sitting activities by sex are described in Table 1 .

There were $6 \%$ of the individuals who reported not exercising any kind of physical activity during their leisure time. Of the sample, $22 \%$ reported a small amount of exercise and the rest $72 \%$ were exercising at least a moderate amount of exercise per year. The amount of leisure-time exercise by sex is described in Table 1. In the preliminary analyses, there was no evidence for differences in physical activity levels regarding the total daily sitting time (data not shown).

In the linear regression analyses, the total sitting time of women was less than sitting time of men (regression 

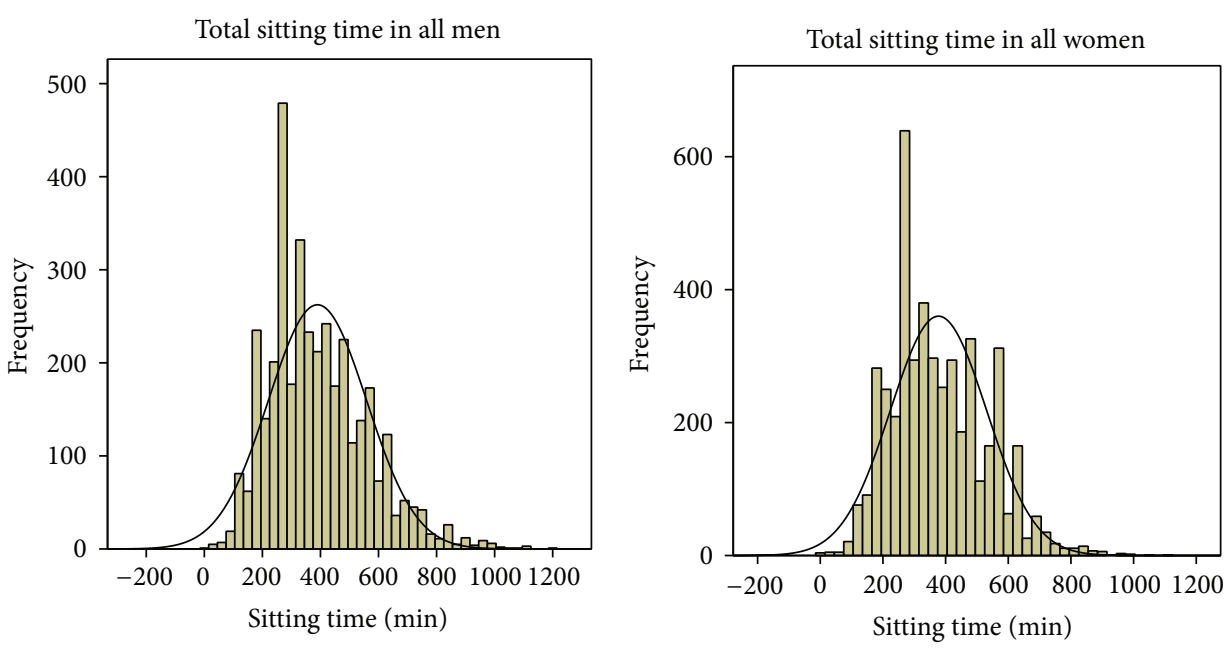

Mean $=389,8$

Std. dev. $=169,612$

$N=3719$

Total sitting time in men (no missing data)

ean $=377,26$

Std. dev. $=153,631$

$N=4623$

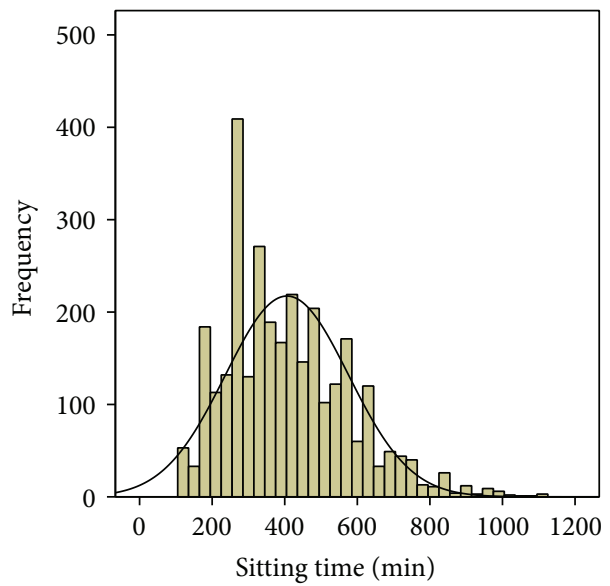

Total sitting time in women (no missing values)

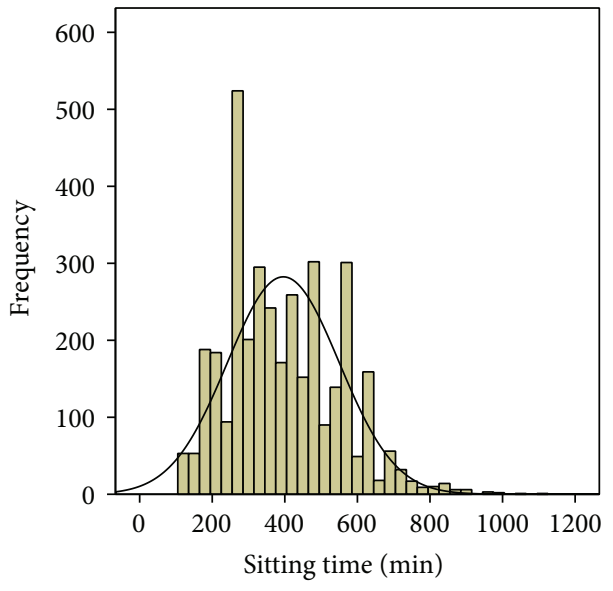

Mean $=406,15$

Std. dev. $=169,692$

$N=3082$

Mean $=395,86$

Std. dev. $=153,862$

$N=3631$

Figure 1: Distribution of total sitting time (minutes) by sex and data included. Total sum score includes all five domains: work, commuting, watching TV, computer use, and others. No missing data includes those with no missing data on any single sitting domain. For those not working, sitting time at work was denoted as zero minutes.

coefficients: -13.01 minutes [95\% CI: $-21.30,-4.83])$ and increase of age decreased the sitting time (regression coefficients: -9.67 minutes per year [95\% CI: $-10.72,-8.63])$. In the analyses of sitting time in different activities (Table 2), sex had no effect on the sitting time at work but sitting at work decreased with age. Both sexes had an equally long sitting time at home watching TV or videos, and age increased this activity. However, men had significantly longer sitting time at the computer at home as they did for sitting time in vehicles compared to women. Increase of age increased computer related sitting time but decreased the amount of vehicle-related sitting time. In other activities, both sexes were sitting an equal amount of time but age increased the sitting time.
As an example of the sedentary behaviour risk factors, the association of body mass index with the total sitting time was analysed. The association of BMI and total time of sitting seemed to be linear in direction that those with higher BMI had also higher total sitting time (regression coefficients 2.78 minutes per BMI unit [95\% CI: 1.77, 3.79], and correlation coefficient 0.064) (Figure 2).

In general, the pairwise correlations of $\mathrm{MZ}$ twins were double compared to correlation of $\mathrm{DZ}$ twins suggesting genetic influences on sedentary behaviour (Table 3). The correlation coefficients were similar for men and women. MZ pairs were more similar for sitting time than DZ pairs, with initial estimates of heritability for the total sitting time being $35 \%$. The influence of shared environmental factors was 
TABLE 1: Domain-based sitting times and the amount of leisure-time physical exercise by sex $\left(n=6713^{*}\right)$.

\begin{tabular}{|c|c|c|}
\hline \multirow{2}{*}{ Activities } & $\operatorname{Men}(n=3082)$ & Women $(n=3631)$ \\
\hline & Mean time (SD) & Mean time (SD) \\
\hline \multicolumn{3}{|l|}{ Sitting time } \\
\hline At work ${ }^{* *}$ & $2 \mathrm{~h} 52 \min (1 \mathrm{~h} 53 \mathrm{~min})$ & $2 \mathrm{~h} 58 \mathrm{~min}(2 \mathrm{~h} 56 \mathrm{~min})$ \\
\hline At home watching TV or videos & $2 \mathrm{~h} 25 \min (1 \mathrm{~h} 12 \mathrm{~min})$ & $2 \mathrm{~h} 25 \mathrm{~min}(1 \mathrm{~h} 11 \mathrm{~min})$ \\
\hline At home at the computer & $57 \mathrm{~min}(52 \mathrm{~min})$ & $50 \mathrm{~min}(43 \mathrm{~min})$ \\
\hline In a vehicle & $59 \min (59 \min )$ & $43 \min (33 \mathrm{~min})$ \\
\hline Elsewhere & $48 \min (45 \min )$ & $48 \min (45 \mathrm{~min})$ \\
\hline \multirow[t]{2}{*}{ Sum of sitting time*** } & $6 \mathrm{~h} 46 \min (2 \mathrm{~h} 50 \mathrm{~min})$ & $6 \mathrm{~h} 36 \mathrm{~min}(2 \mathrm{~h} 34 \mathrm{~min})$ \\
\hline & $n(\%)$ & $n(\%)$ \\
\hline \multicolumn{3}{|c|}{ Amount of leisure-time physical exercise ${ }^{* * * *}$} \\
\hline Practically none & $252(8)$ & $150(4)$ \\
\hline A little & $754(25)$ & $716(20)$ \\
\hline A moderate amount & $1369(45)$ & $1722(48)$ \\
\hline Quite a lot or a great deal & $695(23)$ & $1038(29)$ \\
\hline
\end{tabular}

* Data from those twin individuals who had no missing values in any of the sedentary domains.

** Data from those twin individuals $(n=3970)$ who reported working full time at the moment of the survey.

*** In calculating the total sum of sitting, sitting at office (work) was denoted as zero minutes for those twins $(n=2728)$ who reported not working at the moment of the survey (missing information in 15 individuals).

${ }^{* * * *}$ Missing information in 17 individuals.

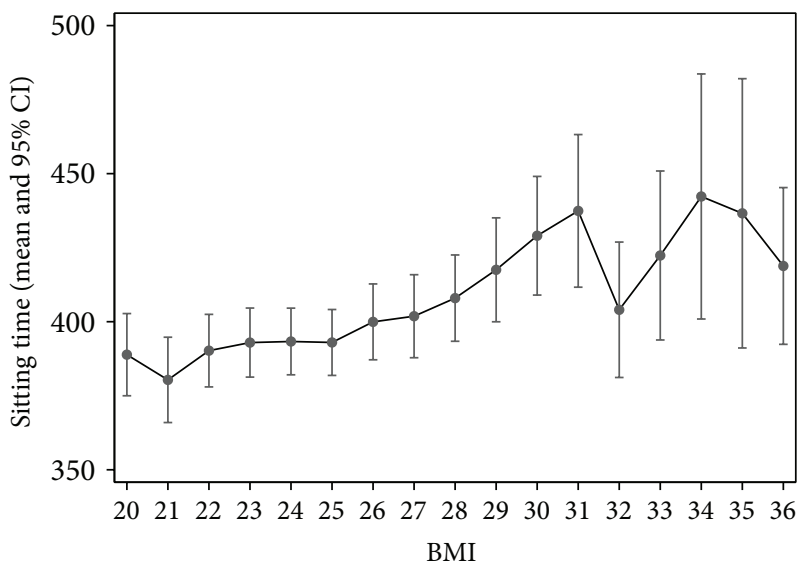

FIGURE 2: Association of BMI and regression model predicted mean of the total sitting time (minutes per day), with $95 \%$ confidence intervals. BMI values 20 or less as well as values 36 or more were combined to be the first and the last categories.

negligible (1\%), while most $(64 \%)$ of the variation could be ascribed to unique environmental factors, the latter including measurement error.

\section{Discussion}

In this sample of 6713 twin individuals, 53-67 years of age, the total amount of sitting was on average 6 hours 41 minutes per day. In a Finnish population-based study of 30-45 years of age, the mean sedentary time (time spent viewing TV,
TABLE 2: Influence of sex and age on sitting time in five different sitting domains in the linear regression model (regression coefficients with $95 \%$ confidence intervals). Men were used as the reference sex.

\begin{tabular}{lccc}
\hline Sitting domain & Coefficient & $95 \%$ CI & $P$ value \\
\hline At work $^{*}$ & & & \\
$\quad$ Female sex & 6.40 & $0.50,12.30$ & 0.034 \\
$\quad$ Age per year & -11.92 & $-12.64,-11.20$ & 0.000 \\
At home watching TV or videos & & & \\
$\quad$ Female sex & 1.10 & $-2.57,4.76$ & 0.558 \\
$\quad$ Age & 2.28 & $1.77,2.79$ & 0.000 \\
At home at the computer & & & \\
$\quad$ Female sex & -6.70 & $-9.13,-4.26$ & 0.000 \\
$\quad$ Age & 0.52 & $0.18,0.87$ & 0.003 \\
In a vehicle & & & \\
$\quad$ Female sex & -16.86 & $-19.31,-14.41$ & 0.000 \\
$\quad$ Age & -1.16 & $-1.46,-0.86$ & 0.000 \\
Elsewhere & & & \\
$\quad$ Female sex & 0.93 & $-1.29,3.14$ & 0.413 \\
$\quad$ Age & 0.74 & $0.44,1.05$ & 0.000 \\
\hline
\end{tabular}

${ }^{*}$ For those twins who were not working at the moment of survey, sitting time at work was denoted as zero minutes $(n=2728)$.

using the computer, reading, listening to music/radio, and in other types of relaxation) was slightly less, a little over 5 hours, [16] but the time spent in vehicles was not inquired into. Another study, involving those of 25-64 years of age, has also shown similar estimates based on the mean selfreported sitting times (including sitting times at work and during leisure time, at home, while visiting friends, studying, 
TABLE 3: Pairwise correlations for sitting time by zygosity.

\begin{tabular}{lcc}
\hline & Number of pairs & Correlation coefficient \\
\hline MZ total & 732 & 0.364 \\
DZ total & 1208 & 0.188 \\
Male MZ & 310 & 0.355 \\
Female MZ & 422 & 0.372 \\
Male DZ & 527 & 0.211 \\
Female DZ & 681 & 0.166 \\
\hline
\end{tabular}

MZ: monozygotic; DZ: dizygotic.

and travelling) during a day: 6.9 hours (SD: 3.5$)$ in men and 6.4 (SD: 3.3) hours in women [15].

In this study, the association between sitting time and age indicated that those of a younger age had higher total sitting time. The association of aging in sedentary behaviour and physical activity patterns is still poorly known [30]. Some evidence exists that younger adults are more active in moderate to vigorous physical activity than older adults [31] but knowledge is lacking on changes in the daily proportion of age-specific sedentary time. Among those with an average 79 years of age, sedentary behaviour explained $24 \%$ of the daily functions [30]. The changes in leisure-time activities after working age can only be speculated. In this study, 59\% of the twin individuals reported working full time during the survey. It is possible that after working age the mean activity level may increase because of more active hobbies and a decreasing amount of sitting in vehicles to and from work, at least for a few years. This hypothesis needs to be studied further with long-term follow-up studies.

Our results also indicate that the increase in total sitting time is associated with increase of BMI. This is in line with other studies where a high amount of TV viewing time has been related to higher BMI and waist circumference $[3,16]$. On the other hand, lower BMI has been related to a higher physical activity level in the aged [30].

There is evidence that genetics has at least a moderate influence on physical activity levels, and age seems to be a regulator of the activity heritability $[21,32,33]$. In adolescents (13-19 years of age), genetic factors explained $72-85 \%$ of the variance in exercise behaviour [20]. In another study, genetics of sedentary behaviour in 12-year-old boys was 35\% and $19 \%$ in girls, whereas the proportion of variance explained by genetics increased to $48 \%$ in 20 -year-old boys and to $34 \%$ in 20-year-old girls, respectively [21]. To the best of our knowledge, our study is among the first ones to study the relative contribution of heritability and environmental factors to sedentary behaviour, measured as total sitting time among older adults. In the present study, the influence of heritability for the total sitting time was 35\%, and most (64\%) of the variation could be ascribed to unique environmental factors. The role of heritability was of equal importance for women and men in this study. The proportion of heritability has been shown to be alike also in a study measuring daily activities with an accelerometer device [23]. However, the genetic component of physical inactivity has reported to be stronger in a comprehensive review [18] compared to our results. It has been reported that the heritability of physical activity decreases with age [33]. However, more sophisticated analyses need to be performed to confirm these results, also using relevant adjusting or stratified variables.

It seems that environmental factors play an important role in sitting time that may be related to adulthood choices or other factors unique to each individual such as occupation and leisure-time activities. The strong influence (50\% to $72 \%$ ) of unique environmental factors on physical activity has also been reported in other twin studies $[22,34]$. These findings suggest that factors influencing our sedentary behaviour should be further elucidated. If the adulthood choices or habits, but also built environments are really of importance for the sitting time, as our preliminary results suggest, there might be possibilities to target public health campaigns to increase the public awareness of sedentary behaviour and/or to target both individual and community-based interventions in order to minimize sedentary behaviour and increase more activity and health enhancing behaviour. Societal policies in urban planning, the work environment, accessing leisuretime facilities, and many others are probably of great importance in their impact on total sitting time. However, we can also explore the role of earlier life circumstances, personality factors, life events, and health status using the cohort data available to us.

One of the main strengths of this study is the large population-based twin data and a high participation rate. The twin study design enables analysing the genetic component of sitting time. Also the generalizability of this twin data should be good since earlier reports have shown that the twins do not differ from the general population in terms of several traits including behaviour [35] or morbidity and mortality [25]. Thus, our study gives new information and aspects in analysing both the prevalence of sedentary behaviour by sitting activity and the relative role of heritability in sitting time.

Recommendations exist that monitoring self-reported sedentary behaviour should include overall sitting time in various activities [11]. In this study, the questionnaire included questions of sitting time in several activities; at work, at home, during commuting, and in all other activities from which we calculated the total time of sitting. Hence, we would like to assume that we have captured well the sitting time during a day. Even though there are both validity and reliability problems in self-rating methods reporting sedentary behaviour [36], there is also evidence that those who are reporting more sedentary behaviour in the selfrating questionnaires are also behaving more sedentary in the objectively measured studies [13]. Previous studies have used predominantly only TV viewing time or TV viewing alongside related "screen time" activities such as computer and video-based time as an indicator of sedentary behaviour $[7,11]$. For example, long TV viewing time has been associated with overweight [3], mortality related to all-causes and cardiovascular diseases [7] as well as mental health [37] independent of many other risk factors or health behaviours. In addition, sitting most of the day has been shown to cause negative effects on insulin sensitivity and plasma lipids [38]. It is, however, noticeable that not all kinds of sitting are harmful 
to our health [37]. Further studies of the age-related sedentary behaviour heritability are needed.

\section{Conclusion}

The amount of sitting time decreases with increasing age but seems to increase along with BMI among older adults. Heritability seems to have a modest role in sitting time with no difference by sex.

\section{Appendices}

\section{A. Questions about Physical Exercise}

What is your daily time of commuting by physically active means (including walking, jogging, cycling, and/or crosscountry skiing) to and from work in total?
(a) less than 15 minutes
(b) 15 minutes-less than half an hour
(c) half an hour-less than an hour
(d) an hour or more
(e) I am not presently at work

Leisure-time physical exercise (exercise that does not occur at work or while commuting to and from work). Here are five alternatives that describe the amount of your leisure-time exercise. Which one applies best to you when considering the amount of exercise you get during the year as a whole?
(a) practically none
(b) a little
(c) a moderate amount
(d) quite a lot
(e) a great deal

How long does one session of your leisure-time physical exercise last an average?
(a) less than 15 minutes
(b) 15 minutes-less than half an hour
(c) half an hour-less than an hour
(d) an hour to less than two hours
(e) over two hours

Presently how many times per month do you engage in physical exercise during your leisure time?
(a) less than once a month
(b) 1-2 times a month
(c) 3-5 times a month
(d) 6-10 times a month
(e) 11-19 times a month
(f) more than 20 times a month

Is your leisure-time physical exercise about as intensive on average as
(a) walking
(b) alternatively walking and jogging
(c) jogging (light run)
(d) running

\section{B. Question about Sitting Time (Sedentary Behaviour)}

How many hours per day do you sit on average?

(1) in office or similar places (e.g., during a working day):
(a) less than an hour
(b) an hour-less than two hours
(c) two hours-less than four hours
(d) four hours or more

(2) at home watching TV or videos:
(a) less than an hour
(b) an hour-less than two hours
(c) two hours-less than four hours
(d) four hours or more

(3) at home at the computer:
(a) less than an hour
(b) an hour-less than two hours
(c) two hours-less than four hours
(d) four hours or more

(4) in a vehicle:
(a) less than an hour
(b) an hour-less than two hours
(c) two hours-less than four hours
(d) four hours or more

(5) elsewhere:
(a) less than an hour
(b) an hour-less than two hours
(c) two hours-less than four hours
(d) four hours or more

\section{Conflict of Interests}

The authors declare that they have no conflict of interests regarding the publication of this paper. 


\section{Acknowledgments}

Data collection was supported by the Academy of Finland Center of Excellence in Complex Disease Genetics (Grant nos. 213506 and 129680 to Jaakko Kaprio) and the Academy of Finland (Grant nos. 265240 and 263278 to Jaakko Kaprio). Analysis was supported by a grant from the Ministry for Education and Culture of Finland to Annina Ropponen. The authors would like to warmly thank the dedicated staff and researchers in our group who took part in the planning and data collection of wave four. A great thanks is due to all the twins who have participated in these studies over the years.

\section{References}

[1] I. M. Lee, E. J. Shiroma, F. Lobelo, P. Puska, S. N. Blair, and P. T. Katzmarzyk, "Effect of physical inactivity on major noncommunicable diseases worldwide: an analysis of burden of disease and life expectancy," The Lancet, vol. 380, no. 9838, pp. 219-229, 2012.

[2] M. T. Hamilton, D. G. Hamilton, and T. W. Zderic, "Role of low energy expenditure and sitting in obesity, metabolic syndrome, type 2 diabetes, and cardiovascular disease," Diabetes, vol. 56, no. 11, pp. 2655-2667, 2007.

[3] S. Inoue, T. Sugiyama, T. Takamiya, K. Oka, N. Owen, and T. Shimomitsu, "Television viewing time is associated with overweight/obesity among older adults, independent of meeting physical activity and health guidelines," Journal of Epidemiology, vol. 22, no. 1, pp. 50-56, 2012.

[4] A. E. Staiano, D. M. Harrington, T. V. Barreira, and P. T. Katzmarzyk, "Sitting time and cardiometabolic risk in US adults: associations by sex, race, socioeconomic status and activity level," British Journal of Sports Medicine, vol. 48, no. 3, pp. 213-219, 2014.

[5] N. Owen, G. N. Healy, C. E. Matthews, and D. W. Dunstan, "Too much sitting: the population health science of sedentary behavior," Exercise and Sport Sciences Reviews, vol. 38, no. 3, pp. 105-113, 2010.

[6] K. I. Proper, A. S. Singh, W. Van Mechelen, and M. J. M. Chinapaw, "Sedentary behaviors and health outcomes among adults: a systematic review of prospective studies," American Journal of Preventive Medicine, vol. 40, no. 2, pp. 174-182, 2011.

[7] Y. Kim, L. R. Wilkens, S. Y. Park, M. T. Goodman, K. R. Monroe, and L. N. Kolonel, "Association between various sedentary behaviours and all-cause, cardiovascular disease and cancer mortality: the Multiethnic Cohort Study," International Journal of Epidemiology, vol. 42, no. 4, pp. 1040-1056, 2013.

[8] H. W. Kohl, C. L. Craig, E. V. Lambert et al., "The pandemic of physical inactivity: global action for public health," The Lancet, vol. 380, no. 9838, pp. 294-305, 2012.

[9] R. R. Pate, J. R. O’Neill, and F. Lobelo, “The evolving definition of 'sedentary"' Exercise and Sport Sciences Reviews, vol. 36, no. 4, pp. 173-178, 2008.

[10] M. Tremblay, "Letter to the editor: standardized use of the terms 'sedentary' and 'sedentary behaviours"' Applied Physiology, Nutrition and Metabolism, vol. 37, no. 3, pp. 540-542, 2012.

[11] G. N. Healy, B. K. Clark, E. A. H. Winkler, P. A. Gardiner, W. J. Brown, and C. E. Matthews, "Measurement of adults' sedentary time in population-based studies," American Journal of Preventive Medicine, vol. 41, no. 2, pp. 216-227, 2011.
[12] H. J. Helmerhorst, S. Brage, J. Warren, H. Besson, and U. Ekelund, "A systematic review of reliability and objective criterion-related validity of physical activity questionnaires," International Journal of Behavioral Nutrition and Physical Activity, vol. 9, p. 103, 2012.

[13] J. M. Schuna Jr., W. D. Johnson, and C. Tudor-Locke, "Adult selfreported and objectively monitored physical activity and sedentary behavior: NHANES 2005-2006," International Journal of Behavioral Nutrition and Physical Activity, vol. 10, no. 1, p. 126, 2013.

[14] A. E. Bauman, R. S. Reis, J. F. Sallis, J. C. Wells, R. J. Loos, and B. W. Martin, "Correlates of physical activity: why are some people physically active and others not?" The Lancet, vol. 380, no. 9838, pp. 258-271, 2012.

[15] K. Borodulin, C. Zimmer, R. Sippola, T. E. Mäkinen, T. Laatikainen, and R. Prättälä, "Health behaviours as mediating pathways between socioeconomic position and body mass index," International Journal of Behavioral Medicine, vol. 19, no. 1, pp. 14-22, 2012.

[16] I. Heinonen, H. Helajärvi, K. Pahkala et al. et al., "Sedentary behaviours and obesity in adults: the Cardiovascular Risk in Young Finns Study," BMJ Open, vol. 3, no. 6, 2013.

[17] D. M. Harrington, T. V. Barreira, A. E. Staiano, and P. T. Katzmarzyk, "The descriptive epidemiology of sitting among US adults, NHANES 2009/2010," Journal of Science and Medicine in Sport, 2013.

[18] D. M. de Vilhena e Santos, P. T. Katzmarzyk, A. F. Seabra, and J. A. Maia, "Genetics of physical activity and physical inactivity in humans," Behavior Genetics, vol. 42, no. 4, pp. 559-578, 2012.

[19] J. H. Stubbe, D. I. Boomsma, J. M. Vink et al., "Genetic influences on exercise participation in 37.051 twin pairs from seven countries," PLoS ONE, vol. 1, no. 1, article e22, 2006.

[20] N. van der Aa, E. J. De Geus, T. C. van Beijsterveldt, D. I. Boomsma, and M. Bartels, "Genetic influences on individual differences in exercise behavior during adolescence," International Journal of Pediatrics, vol. 2010, Article ID 138345, 8 pages, 2010.

[21] N. van der Aa, M. Bartels, S. J. te Velde, D. I. Boomsma, E. J. de Geus, and J. Brug, "Genetic and environmental influences on individual differences in sedentary behavior during adolescence: a twin-family study," Archives of Pediatrics and Adolescent Medicine, vol. 166, no. 6, pp. 509-514, 2012.

[22] M. C. Nelson, P. Gordon-Larsen, K. E. North, and L. S. Adair, "Body mass index gain, fast food, and physical activity: effects of shared environments over time," Obesity, vol. 14, no. 4, pp. 701-709, 2006.

[23] M. den Hoed, S. Brage, J. H. Zhao et al., "Heritability of objectively assessed daily physical activity and sedentary behavior," The American Journal of Clinical Nutrition, 2013.

[24] J. Kaprio and M. Koskenvuo, "Genetic and environmental factors in complex diseases: the older Finnish Twin Cohort," Twin Research, vol. 5, no. 5, pp. 358-365, 2002.

[25] J. Kaprio, “The Finnish Twin Cohort Study: an update," Twin Research and Human Genetics, vol. 16, no. 1, pp. 157-162, 2013.

[26] J. Kaprio, M. Koskenvuo, and S. Sarna, "Cigarette smoking, use of alcohol, and leisure-time physical activity among same-sexed adult male twins," Progress in clinical and biological research, vol. 69, pp. 37-46, 1981.

[27] T. Lumley, P. Diehr, S. Emerson, and L. Chen, "The importance of the normality assumption in large public health data sets," Annual Review of Public Health, vol. 23, pp. 151-169, 2002. 
[28] R. L. Williams, "A note on robust variance estimation for clustercorrelated data," Biometrics, vol. 56, no. 2, pp. 645-646, 2000.

[29] D. S. Falconer and T. F. C. Mackay, Introduction To Quantitative Genetics, Longmans Green, Essex, UK, 4th edition, 1996.

[30] S. Lord, S. F. M. Chastin, L. McInnes, L. Little, P. Briggs, and L. Rochester, "Exploring patterns of daily physical and sedentary behaviour in community-dwelling older adults," Age and Ageing, vol. 40, no. 2, Article ID afq166, pp. 205-210, 2011.

[31] M. G. Davis and K. R. Fox, "Physical activity patterns assessed by accelerometry in older people," European Journal of Applied Physiology, vol. 100, no. 5, pp. 581-589, 2007.

[32] T. Moore-Harrison and J. T. Lightfoot, "Driven to be inactive? The genetics of physical activity," Progress in molecular biology and translational science, vol. 94, pp. 271-290, 2010.

[33] J. M. Vink, D. I. Boomsma, S. E. Medland et al., "Variance components models for physical activity with age as modifier: a comparative twin study in seven countries," Twin Research and Human Genetics, vol. 14, no. 1, pp. 25-34, 2011.

[34] G. E. Duncan, J. Goldberg, C. Noonan, A. V. Moudon, P. Hurvitz, and D. Buchwald, "Unique environmental effects on physical activity participation: a twin study," PLoS ONE, vol. 3, no. 4, Article ID e2019, 2008.

[35] L. Pulkkinen, I. Vaalamo, R. Hietala, J. Kaprio, and R. J. Rose, "Peer reports of adaptive behavior in twins and singletons: is twinship a risk or an advantage?" Twin Research, vol. 6, no. 2, pp. 106-118, 2003.

[36] B. K. Clark, T. Sugiyama, G. N. Healy, J. Salmon, D. W. Dunstan, and N. Owen, "Validity and reliability of measures of television viewing time and other non-occupational sedentary behaviour of adults: a review," Obesity Reviews, vol. 10, no. 1, pp. 7-16, 2009.

[37] M. Hamer and E. Stamatakis, "Prospective study of sedentary behavior, risk of depression, and cognitive impairment," Medicine and Science in Sports and Exercise, vol. 46, no. 4, pp. 718-723, 2014.

[38] B. M. Duvivier, N. C. Schaper, M. A. Bremers et al., "Minimal intensity physical activity (standing and walking) of longer duration improves insulin action and plasma lipids more than shorter periods of moderate to vigorous exercise (cycling) in sedentary subjects when energy expenditure is comparable," PLoS ONE, vol. 8, no. 2, Article ID e55542, 2013. 

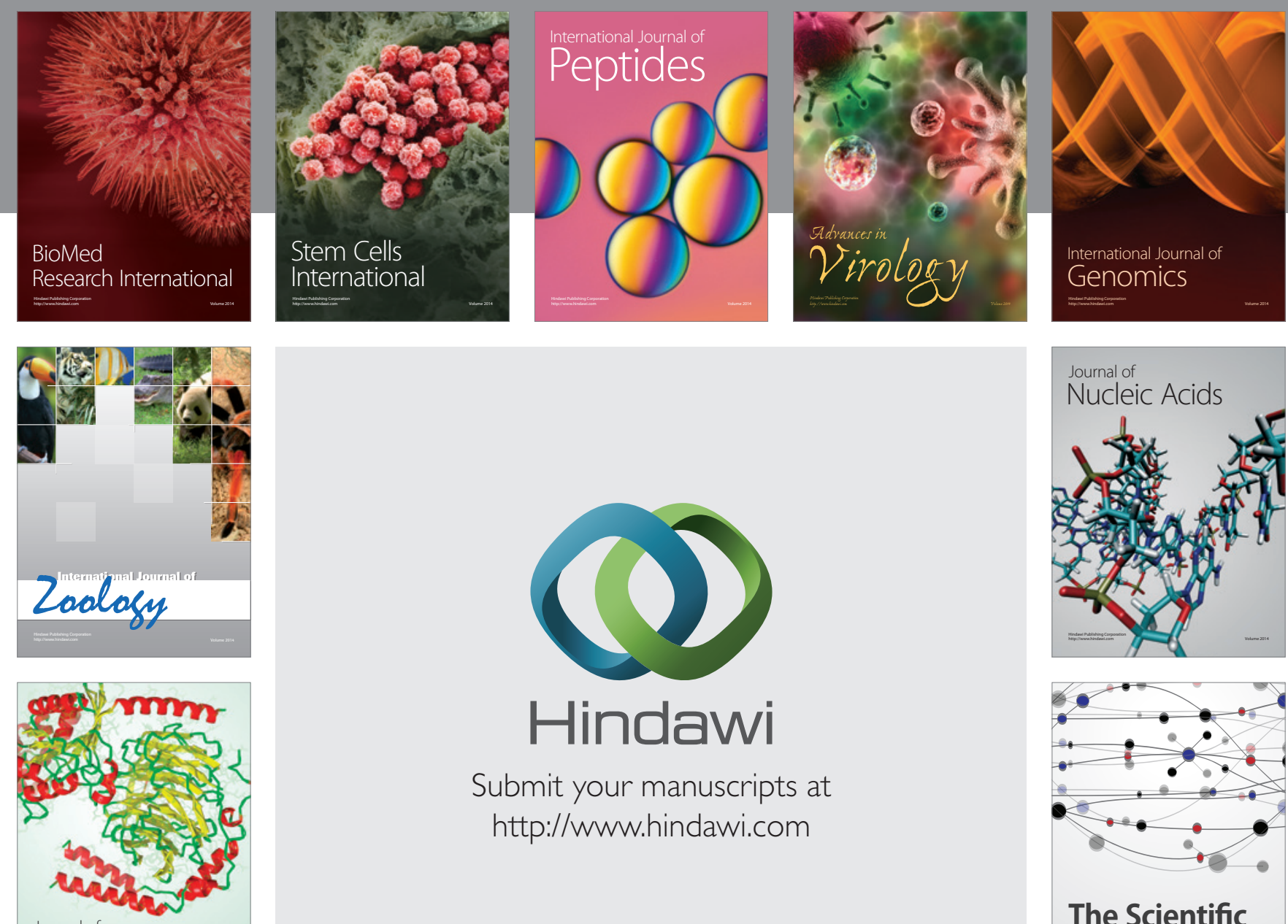

Submit your manuscripts at

http://www.hindawi.com

Journal of
Signal Transduction
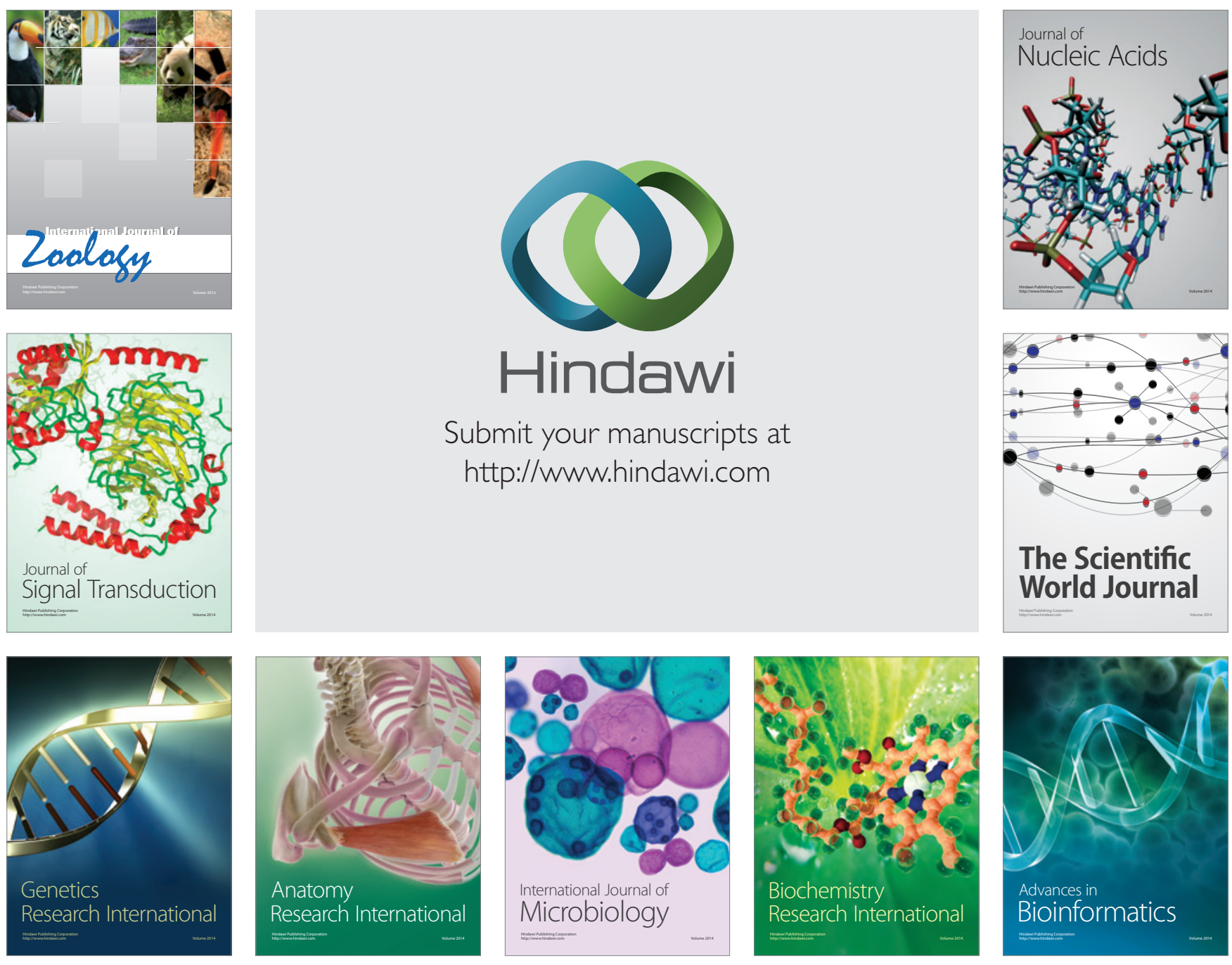

The Scientific World Journal
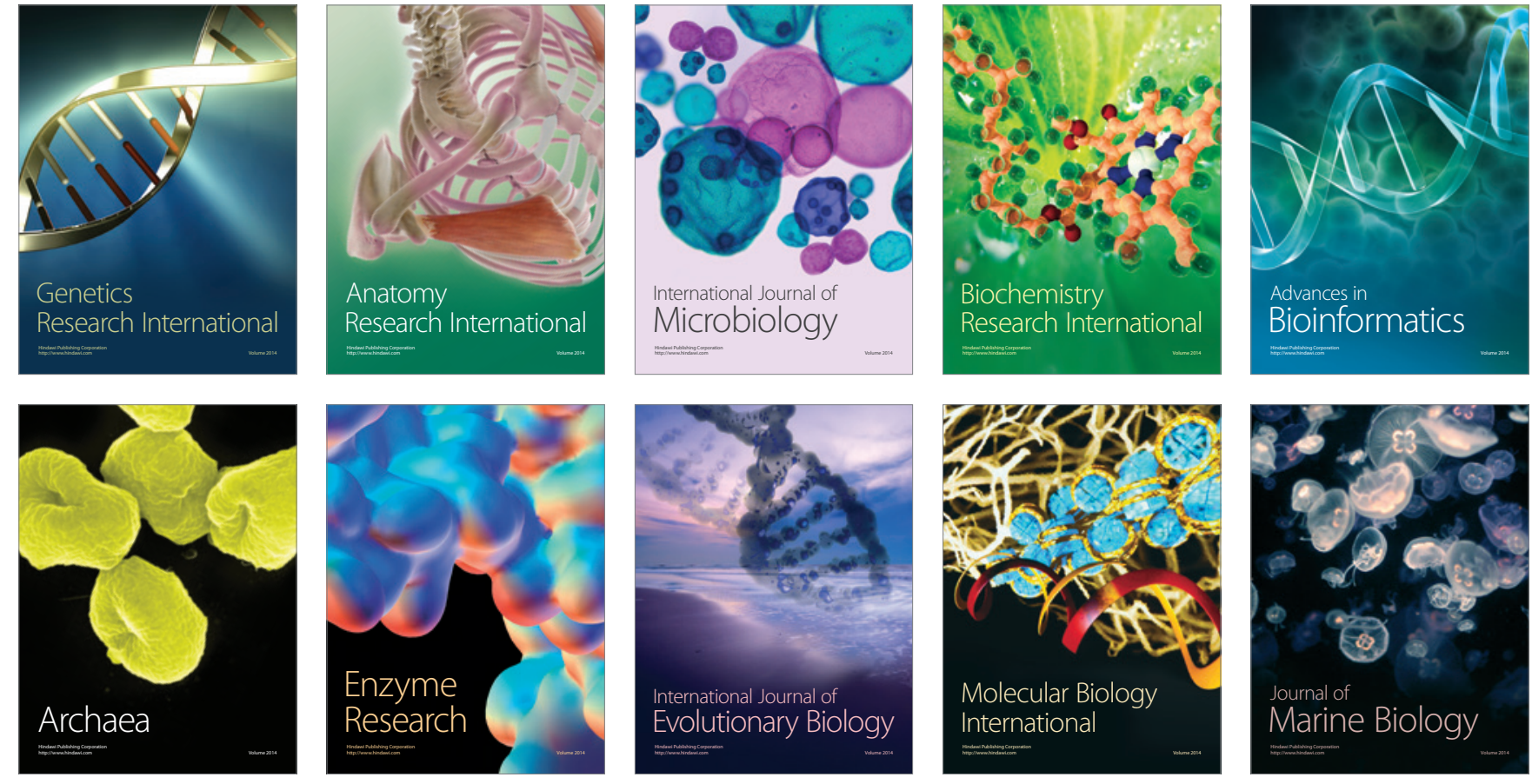